\title{
Dynamic characteristics of spherically converging detonation waves
}

\author{
Zonglin Jiang · Lina Chang · Fan Zhang
}

Received: 30 March 2005 / Accepted: 14 November 2006 / Published online: 6 January 2007

(C) Springer-Verlag 2006

\begin{abstract}
The spherically converging detonation wave was numerically investigated by solving the one-dimensional multi-component Euler equations in spherical coordinates with a dispersion-controlled dissipative scheme. Finite rate and detailed chemical reaction models were used and numerical solutions were obtained for both a spherical by converging detonation in a stoichiometric hydrogen-oxygen mixture and a spherically focusing shock in air. The results showed that the post-shock pressure approximately arises to the same amplitude in vicinity of the focal point for the two cases, but the post-shock temperature level mainly depends on chemical reactions and molecular dissociations of a gas mixture. While the chemical reaction heat plays an important role in the early stage of detonation wave propagation, gas dissociations dramatically affect the post-shock flow states near the focal point. The maximum pressure and temperature, non-dimensionalized by their initial value, are approximately scaled to the propagation radius over the initial detonation diameter. The post-shock pressure is proportional to the initial
\end{abstract}

Communicated by K. Takayama.

Zonglin Jiang is presently a visiting professor at McGill University, Canada.

Z. Jiang $(\varangle) \cdot$ L. Chang

Key Laboratory of High Temperature Gas Dynamics,

Chinese Academy of Sciences, Institute of Mechanics,

15 Zhong Guan Cun Road, Beijing 100080, China

e-mail: zljiang@imech.ac.cn

F. Zhang

Defence Research and Development Canada-Suffield,

P.O. Box 4000 Stn. Main, Medicine Hat,

AB T1A 8K6 Canada pressure of the detonable mixture, and the post-shock temperature is also increased with the initial pressure, but in a much lower rate than that of the post-shock pressure.

Keywords Spherically converging detonation . Spherical shock wave · Gas dissociation - Chemical reaction $\cdot$ Numerical simulation

PACS 47.40.-x $\cdot$ 47.40.Rs $\cdot 47.70 . \mathrm{Fw}$

\section{Introduction}

The spherically converging detonation wave has intrigued researchers for decades due to its inherent implosion ability. The converging geometry forces the detonation wave front into an ever-decreasing area that creates additional compression as compared with that of planar geometries. The end result is a positive feedback cycle, i.e., the increasing compression accelerates the overdriven detonation, which, in turn, acts to further increase the post-detonation pressure and temperature. This cycle continues during the converging process and can result in an overdriven detonation with extremely high post-detonation pressures and temperatures. However, it remains unresolved whether and when a spherical detonation wave would reach a limit due to endothermic dissociations as it converges towards its center.

The spherically diverging detonation and its analytic solutions in a quasi-steady state were studied by a number of researchers. Detailed information can be found in literature, eg., in the review by Lee et al. [1] and Nettleton [2]. Kogarko et al. [3] experimentally 
investigated spherically diverging detonation in a methane/air mixture using a strong overdriven initiation by a solid explosive charge. In their study, the wave propagation distance was insufficient to exclude the influence of the strong initiation on the observed behavior. Bull et al. [6] reported an experimental study on spherical detonation waves in methane/oxygen/nitrogen mixtures, using various masses of solid explosive (Tetryl) as an initiator. They established the detonation limit at the atmospheric pressure and the measured detonation velocities were all close to calculated Chapman-Jouguet (CJ) velocities.

On the spherically converging detonation, analytical investigations have been carried out since 1950s. The method for analyzing planar detonation waves was used to get approximate solutions for the spherical detonations in its earlier converging stage. As the detonation converges, the additional compression is created due to a decrease in the wave front area, thus resulting in difficulties for a solution due to effects of both chemical reaction and geometry compression. As the wave front approaches to its focal point, the detonation becomes strongly overdriven and the effect of the chemical energy release could become negligible. As a result, the detonation wave in the vicinity of the focus point might be treated as a strong shock imploding. The similarity solution proposed by Guderley [3] for imploding shock waves could therefore be applied in the later stage of the spherically converging detonation. However, this solution provided only a rough estimate by neglecting endothermic reactions behind the strong shock front near the focal point. In 1950s, the CCW relation had developed by Chester, Chisnell, and Whitham independently, to describe the shock wave imploding in a homogeneous gas. Li et al. [4] modified the CCW relation to take chemical heat release into account and applied it to a converging detonation wave. This modified CCW theory did not consider the heat release rate and gas dissociations associated with the ever-increasing postshock temperature and therefore was limited for the application near the focal point.

Experimentally, it is challenging to generate a spherically converging detonation using synchronous initiation around a sphere. It is even more difficult to measure the post-detonation flow state due to extremely high parameters and resolution near the focal point. Hence, few experimental studies have been available in the open literature for the spherically converging detonation. Terao et al. [7,8] carried out experiments on a spherically converging detonation in a stoichiometric propane-oxygen mixture at an initial pressure of $40 \mathrm{kPa}$ and a room temperature of $293 \mathrm{~K}$. Their experimental results indicated that an extremely high temperature between $10^{7}$ and $10^{8} \mathrm{~K}$ was observed within the implosion focus of $0.5 \mathrm{~mm}$ in diameter in an initial convergent space having a diameter of $500 \mathrm{~mm}$.

It is known from both the modified CCW model and experiments that a spherically imploding detonation wave accelerates much slowly towards the focus than a nonreactive shock wave of the same strength. To our knowledge, there have been few attempts to determine the evolution of a converging detonation front initiated at the $\mathrm{CJ}$ conditions propagating towards the center of convergence, and to establish the role of chemical reaction and gas dissociations in the converging process. The objective of the present work is to numerically study a spherically converging detonation wave in order to identify its dynamic characteristics at various initial and geometric conditions. A hydrogen/oxygen mixture is modeled using 20 elementary chemical reactions involving nine species. The chemical reactions and dissociations, which cannot be modeled appropriately in analytical approaches, are examined here in details. In order to determine the role of the gas dissociations, the spherical shock wave focusing in air is also investigated, where the air is treated as a gas mixture of oxygen and nitrogen, including five species and 17 elementary chemical reactions. In numerical simulations, the one-dimensional multi-component Euler equations in spherical coordinates are solved by using a dispersion-controlled dissipative scheme. A polynomial function suggested by NASA Glenn research center is applied to calculate the thermodynamic parameters of reactive mixtures [11]. In order to verify the CFD scheme, the spherically diverging and the planar detonation waves were first calculated and compared with the experiments found in literatures. The CFD scehme is then used to study the spherically converging detonation and the spherical shock focusing. Variations of the pressure and temperature profiles behind the wave front are traced step by step during the simulations, and their dependence on the detonation geometrical sizes, initial conditions, the chemical reaction and dissociation is discussed.

\section{Governing equations and chemical reaction model}

Assuming that the effect of viscosity on the dynamic process of a converging shock wave and detonation wave is negligible and the flow field of interest is of spherical symmetry, the governing equations for spherically converging detonations can be simplified as the onedimensional multi-components Euler equations with the source terms including chemical reactions. The equations can be written in conservation form with $n_{s}$ inhomogeneous continuity equations for an ideal gas in 
spherical coordinates:

$\frac{\partial U}{\partial \tau}+\frac{\partial F}{\partial r}=S$

where

$U=r^{2}\left(\rho_{1}, \rho_{2}, \ldots, \rho_{n_{s}}, \rho v, E\right)^{t}$

$F=r^{2}\left(\rho_{1} v, \rho_{2} v, \ldots, \rho_{n_{s}} v, \rho v^{2}+p,(E+p) v\right)^{t}$

$S=r^{2}\left(\dot{\omega}_{1}, \dot{\omega}_{2}, \ldots, \dot{\omega}_{n_{s}}, 2 p / r, 0\right)^{t}$

where $U, F, S, \tau$, and $r$ are unknown variables, flux, source terms, time and space coordinate, respectively. The density of each species is denoted by $\rho_{i}$, and $\rho=$ $\sum_{i=1}^{n_{s}} \rho_{i}$ is the density of the gas mixture. $v$ is velocity, and $p$ is the gas mixture pressure given by $p=\sum_{i=1}^{n_{s}} \rho_{i} R_{i} T$ according to Dalton's law. $E$ is the total energy of the gas mixture per unit volume, $n_{S}$ is the number of species being present in chemical reaction. In this paper, the following polynomial expression is employed to calculate the constant pressure specific heat of each individual species.

$$
\begin{aligned}
\frac{c_{p i}}{R_{i}}= & a_{1 i} \frac{1}{T^{2}}+a_{2 i} \frac{1}{T}+a_{3 i}+a_{4 i} T \\
& +a_{5 i} T^{2}+a_{6 i} T^{3}+a_{7 i} T^{4}
\end{aligned}
$$

here, $T$ is the temperature and $R_{i}$ is the gas constant for species $i$. The enthalpy per unit mass for each species is written as:

$$
\begin{aligned}
\frac{h_{i}}{R_{i} T}= & -a_{1 i} \frac{1}{T^{2}}+a_{2 i} \frac{1}{T} \ln T+a_{3 i}+\frac{a_{4 i}}{2} T \\
& +\frac{a_{5 i}}{3} T^{2}+\frac{a_{6 i}}{4} a_{6 i} T^{3}+\frac{a_{7 i}}{5} T^{4}+\frac{b_{1 i}}{T}
\end{aligned}
$$

The total enthalpy per unit mass for the gas mixture is denoted by $h$, and given by $h=\sum_{i=1}^{n_{s}} Y_{i} h_{i}$, where $Y_{i}$ is mass fraction. One can write $E=e+\rho v^{2} / 2$, where $e$ is the internal energy per unit volume, given by $e=$ $\rho h-p$. The coefficients $a_{1 i}, a_{2 i}, \ldots, a_{7 i}$ and $b_{1 i}$ are given in Ref. [6].

The source terms, $\dot{\omega}_{i}$, for $i=1,2, \ldots, n_{s}$, in Eq. 1 are the summation of the net rate change of species $i$ from all chemical reactions involved, i.e.

$\dot{\omega}_{i}=W_{i} \sum_{k=1}^{n_{r}}\left(v_{i k}^{\prime \prime}-v_{i k}^{\prime}\right)\left(K_{f k} \prod_{j=1}^{n_{s}}\left(C_{j}\right)^{v_{j k}^{\prime}}-K_{b k} \prod_{j=1}^{n_{s}}\left(C_{j}\right)^{v_{j k}^{\prime \prime}}\right)$

where $W_{i}$ is molecular weight of species $i . n_{r}$ is the number of chemical reactions being considered in detonation. $v_{i k}^{\prime}$ and $v_{i k}^{\prime \prime}$ are stoichiometric coefficients of reactants and products, respectively, for species $i$ in the $k$ th reaction. $C_{j}$ is the mole concentration of species $j$. The forward reaction rate constant, $K_{f, k}$, is determined by the Arrhenius equation. Table 1 gives the Arrhenius parameters for the hydrogen-oxygen mixture and Table 2 shows these for air dissociation. $K_{b, k}$ is the backward reaction rate constant, given by $K_{b, k}=K_{f, k} / K_{c, k}$,

\begin{tabular}{|c|c|c|c|c|}
\hline No. & Reaction equation & $A$ & $n$ & $E a$ \\
\hline 1 & $\mathrm{H}_{2}+\mathrm{O}_{2}=2 \mathrm{OH}$ & $1.70 \times 10^{13}$ & 0.0 & 47,780 \\
\hline 2 & $\mathrm{OH}+\mathrm{H}_{2}=\mathrm{H}_{2} \mathrm{O}+\mathrm{H}$ & $1.17 \times 10^{9}$ & 1.3 & 3,626 \\
\hline 3 & $\mathrm{O}+\mathrm{OH}=\mathrm{O}_{2}+\mathrm{H}$ & $4.00 \times 10^{14}$ & -0.5 & 0 \\
\hline 4 & $O+H_{2}=O H+H$ & $5.06 \times 10^{4}$ & 2.7 & 6,290 \\
\hline 5 & $H+\mathrm{O}_{2}+\mathrm{M}=\mathrm{HO}_{2}+\mathrm{M}$ & $3.61 \times 10^{17}$ & -0.7 & 0 \\
\hline 6 & $\mathrm{OH}+\mathrm{HO}_{2}=\mathrm{H}_{2} \mathrm{O}+\mathrm{O}_{2}$ & $7.50 \times 10^{12}$ & 0.0 & 0 \\
\hline 7 & $\mathrm{H}+\mathrm{HO}_{2}=2 \mathrm{OH}$ & $1.40 \times 10^{14}$ & 0.0 & 1,073 \\
\hline 8 & $\mathrm{O}+\mathrm{HO}_{2}=\mathrm{O}_{2}+\mathrm{OH}$ & $1.40 \times 10^{13}$ & 0.0 & 1,073 \\
\hline 9 & $2 \mathrm{OH}=\mathrm{O}+\mathrm{H}_{2} \mathrm{O}$ & $6.00 \times 10^{8}$ & 1.3 & 0 \\
\hline 10 & $H+H+M=H_{2}+M$ & $1.00 \times 10^{18}$ & -1.0 & 0 \\
\hline 11 & $H+H+H_{2}=H_{2}+H_{2}$ & $9.20 \times 10^{16}$ & -0.6 & 0 \\
\hline 12 & $\mathrm{H}+\mathrm{H}+\mathrm{H}_{2} \mathrm{O}=\mathrm{H}_{2}+\mathrm{H}_{2} \mathrm{O}$ & $6.00 \times 10^{19}$ & -1.3 & 0 \\
\hline 13 & $\mathrm{H}+\mathrm{OH}+\mathrm{M}=\mathrm{H}_{2} \mathrm{O}+\mathrm{M}$ & $1.60 \times 10^{22}$ & -2.0 & 0 \\
\hline 14 & $H+O+M=O H+M$ & $6.20 \times 10^{16}$ & -0.6 & 0 \\
\hline 15 & $O+O+M=O_{2}+M$ & $1.89 \times 10^{13}$ & 0.0 & $-1,788$ \\
\hline 16 & $\mathrm{H}+\mathrm{HO}_{2}=\mathrm{H}_{2}+\mathrm{O}_{2}$ & $1.25 \times 10^{13}$ & 0.0 & 0 \\
\hline 17 & $\mathrm{HO}_{2}+\mathrm{HO}_{2}=\mathrm{H}_{2} \mathrm{O}_{2}+\mathrm{O}_{2}$ & $2.00 \times 10^{12}$ & 0.0 & 0 \\
\hline 18 & $\mathrm{H}_{2} \mathrm{O}_{2}+\mathrm{M}=2 \mathrm{OH}+\mathrm{M}$ & $1.30 \times 10^{17}$ & 0.0 & 45,500 \\
\hline 19 & $\mathrm{H}_{2} \mathrm{O}_{2}+\mathrm{H}=\mathrm{HO}_{2}+\mathrm{H}_{2}$ & $1.60 \times 10^{12}$ & 0.0 & 3,800 \\
\hline 20 & $\mathrm{H}_{2} \mathrm{O}_{2}+\mathrm{OH}=\mathrm{H}_{2} \mathrm{O}+\mathrm{HO}_{2}$ & $1.00 \times 10^{13}$ & 0.0 & 1,800 \\
\hline
\end{tabular}
and $K_{c, k}$ is the equilibrium constant for the chemical reaction.

Table 1 Reaction mechanism for hydrogen-oxygen detonation

considered species: $\mathrm{H}_{2}, \mathrm{O}_{2}, \mathrm{H}_{2} \mathrm{O}, \mathrm{H}, \mathrm{OH}, \mathrm{HO}_{2}, \mathrm{H}_{2} \mathrm{O}_{2}, \mathrm{O} . \quad k_{f}=$ $A T^{n} e^{-E_{a} / R T}$, parameters are given in mole, $\mathrm{s}, \mathrm{cm}, \mathrm{K}$ and kal. Third body coefficients: Reaction 5: $\mathrm{H}_{2} \mathrm{O}=18.6, \mathrm{H}_{2}=2.86$, Reaction, 10: $\mathrm{H}_{2} \mathrm{O}=0, \mathrm{H}_{2}=0$, Reaction, 13 and 14: $\mathrm{H}_{2} \mathrm{O}=5.0$

Table 2 Reaction mechanism of air dissociation

\begin{tabular}{rllrl}
\hline No & Reaction equation & $A$ & $\mathrm{n}$ & $E a$ \\
\hline 1 & $O_{2}+N=O+O+N$ & $1.0 \times 10^{22}$ & -1.5 & 494.7 \\
2 & $O_{2}+O=O+O+O$ & $1.0 \times 10^{22}$ & -1.5 & 494.7 \\
3 & $O_{2}+N_{2}=O+O+N_{2}$ & $2.0 \times 10^{21}$ & -1.5 & 494.7 \\
4 & $O_{2}+O_{2}=O+O+O_{2}$ & $2.0 \times 10^{21}$ & -1.5 & 494.7 \\
5 & $O_{2}+N O=O+O+N O$ & $2.0 \times 10^{21}$ & -1.5 & 494.7 \\
6 & $N_{2}+N=N+N+N$ & $3.0 \times 10^{22}$ & -1.6 & 941.2 \\
7 & $N_{2}+O=N+N+O$ & $3.0 \times 10^{22}$ & -1.6 & 941.2 \\
8 & $N_{2}+N_{2}=N+N+N_{2}$ & $7.0 \times 10^{21}$ & -1.6 & 941.2 \\
9 & $N_{2}+O_{2}=N+N+O_{2}$ & $7.0 \times 10^{21}$ & -1.6 & 941.2 \\
10 & $N_{2}+N O=N+N+N O$ & $7.0 \times 10^{21}$ & -1.6 & 941.2 \\
11 & $N O+N=N+O+N$ & $1.1 \times 10^{17}$ & 0.0 & 627.7 \\
12 & $N O+O=N+O+O$ & $1.1 \times 10^{17}$ & 0.0 & 627.7 \\
13 & $N O+N_{2}=N+O+N_{2}$ & $5.0 \times 10^{15}$ & 0.0 & 627.7 \\
14 & $N O+O 2=N+O+O_{2}$ & $5.0 \times 10^{15}$ & 0.0 & 627.7 \\
15 & $N O+N O=N+O+N O$ & $1.1 \times 10^{17}$ & 0.0 & 627.7 \\
16 & $N_{2}+O=N O+N$ & $1.8 \times 10^{14}$ & 0.0 & 318.4 \\
17 & $O+N O=O+N$ & $6.9 \times 10^{8}$ & 1.1 & 159.6 \\
\hline
\end{tabular}

considered species: $O_{2}, N_{2}, N O, O, N . k_{f}=A T^{n} e^{-E_{a} / R T}$, parameters are given in mole, $\mathrm{s}, \mathrm{cm}, \mathrm{K}$ and $\mathrm{kJ}$ 
The model involving 20-elementary reactions of nine species is accepted to account for hydrogen-oxygen combustion. The reacting species are $\mathrm{H}_{2}, \mathrm{O}_{2}, \mathrm{H}, \mathrm{O}, \mathrm{OH}$, $\mathrm{H}_{2}, \mathrm{O}_{2}, \mathrm{HO}_{2}$ and $\mathrm{H}_{2} \mathrm{O}$. A certain percentage of argon diluent is added to the gas mixture. This gas mixture is selected for the numerical simulation because extensive results are available for comparison and verification (e.g., Schultz et al. [13]; Chang et al. [15]).

\section{Numerical algorithm}

For numerical simulations, the second-order explicit difference equations of Eq. 1 discretized in space by using the dispersion-controlled dissipative scheme proposed by Jiang are written as $[9,10]$ :

$$
\begin{aligned}
& U_{j}^{n+1}=U_{j}^{n}-\frac{1}{r_{j}^{2}} \frac{\Delta t}{\Delta r}\left(Q_{j+\frac{1}{2}}^{n}-Q_{j-\frac{1}{2}}^{n}\right) \\
& \left\{\begin{array}{l}
Q_{j+\frac{1}{2}}=r_{j+\frac{1}{2}}^{2}\left(F_{j+\frac{1}{2}, L}^{+}+F_{j+\frac{1}{2}, R}^{-}\right) \\
F_{j+\frac{1}{2}, L}^{+}=F_{j}^{+}+\frac{1}{2} \operatorname{minmod}\left(\Delta F_{j-\frac{1}{2}}^{+}, \Delta F_{j+\frac{1}{2}}^{-}\right) \\
F_{j+\frac{1}{2}, R}^{-}=F_{j+1}^{-}-\frac{1}{2} \operatorname{minmod}\left(\Delta F_{j+\frac{1}{2}}^{+}, \Delta F_{j+\frac{3}{2}}^{-}\right)
\end{array}\right.
\end{aligned}
$$

where

$$
\Delta F_{j+\frac{1}{2}}^{ \pm}=F_{j+1}^{ \pm}-F_{j}^{ \pm}
$$

In the above equations, $(\cdot)^{+}$and $(\cdot)^{-}$superscript signs denote the flux vector splitting according to the Steger and Warming method [12]. As to the dispersion-controlled dissipative scheme, it was proposed based on the dispersion conditions derived by considering the role of dispersion terms in the modified equation proposed by Warming and Hyett. The conditions require that shockcapturing schemes must be of different phrase errors (leading or lagging) to avoid non-physical oscillations when calculating the fluid flow at each side of shock waves [9]. By using the scheme, non-oscillatory solutions can be achieved without any need for artificial viscosity and free parameters. Such a characteristic is very helpful to highlight the shock wave, which may, otherwise, be smeared with the artificial viscosity.

In computation, the stiff problem due to chemical reactions is solved by using a splitting algorithm, that is, at each time step in numerical simulations, the contribution of fluid dynamic terms to next flow states is calculated first to obtain an intermediate value of $U^{\prime}$, and then this is followed by accounting for the contribution from chemical reactions to evaluate $U$ from the intermediate value. The numerical approach allows to separately calculate the fluid dynamic terms and the source terms containing chemical reaction with different time steps. The implication is that the fluid dynamic terms can be integrated during $\Delta t$ being consistent with the $C F L$ condition, and the source terms are calculated by using a much smaller time step required by the chemical reaction time scale. This treatment works well for the present detonation calculation.

\section{Problem specifications}

\subsection{Problem descriptions}

The computational domain, boundary and initial conditions for the spherically converging detonation are schematically shown in Fig. 1, where the C-J planar detonation was initially specified at a distance of $R$ from the center of the domain and is to propagate toward the center in the gas mixture of the initial states as indicated in the figure. $R$ is the initial diameter of the spherical detonation front, by which the effect of ever-decreasing space on detonation can considered. Assuming that the converging effect on the post-detonation flow states is negligible for a sufficiently large $R$ at the beginning of the detonation converging, the planar detonation calculated based on the initial state can be accepted as initial conditions. In doing so, the effect of detonation ignition on the converging process can be removed. The computation will be terminated when the detonation front propagates near the focal point and reaches a maximum temperature of $7,000 \mathrm{~K}$. Beyond this temperature limit, the present chemical reaction model cannot be used for appropriate prediction and would result in extremely high temperature. Therefore, the present study will focus on dynamic characteristics of the spherical detonation wave during its converging, but not on the final imploding flow states.

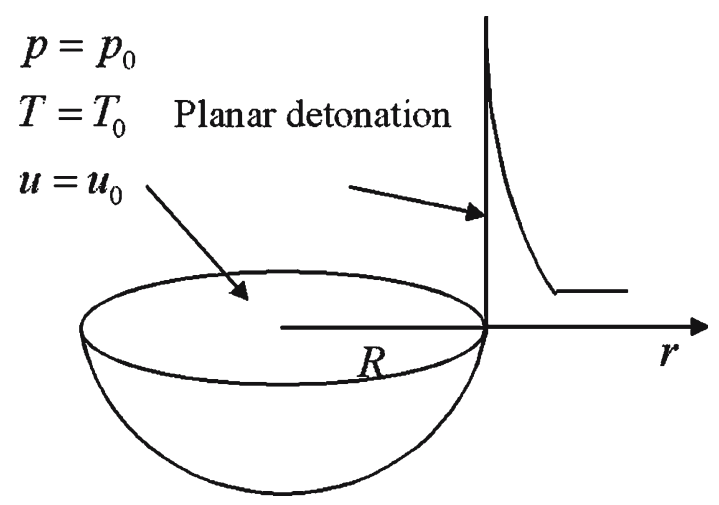

Fig. 1 Computational domain and initial conditions for spherically converging detonation 


\subsection{Code verification}

In order to verify the models and the numerical code, two cases were calculated. The first case is a planar detonation propagating in a straight tube to validate the applied chemical reaction model, and the second case is a spherically diverging detonation to validate the onedimensional governing equations. The gaseous mixture is the stoichiometric hydrogen/oxygen with $20 \%$ argon dilution at initial conditions of $P_{0}=20 \mathrm{kPa}$ and $T_{0}=$ $295 \mathrm{~K}$. Some detonation parameters from the numerical results are summarized in Table 3 for comparison.

Table 3 shows the $\mathrm{C}-\mathrm{J}$ parameters of two cases, including the detonation propagation speed, the detonation pressure and temperature. From the table, it can be seen that the present results of the planar detonation are slightly smaller than the ones calculated by Schultz and Shepherd [13] with the ZND model. The discrepancies in the $\mathrm{C}-\mathrm{J}$ speed and temperature are within $1 \%$, and these in the $\mathrm{C}-\mathrm{J}$ pressure are bigger, but still falls in a $6 \%$ limitation. By comparing the planar case with the spherical one, one could see that the spherically diverging detonation is weaker than the planar one. This is believed due to stronger expansion waves resulting from ever-expanding detonation geometry.

The present numerical results are also compared with the theoretical prediction using the Taylor self-similarity law, as displayed in Fig. 2. The spherically diverging case is shown in Fig. 2a and the planar one is in Fig. 2b. Generally speaking, the numerical pressure and velocity profiles are in good agreement with the theoretical prediction, that is, the $\mathrm{C}-\mathrm{J}$ plan following by a series of rarefaction waves, and then a uniform column of the slug flow at rest to meet the zero-velocity boundary condition. However, there exist some notable discrepancies in their values. The velocity distribution of the numerical results is a bit lower and the pressure is slightly higher than the theoretical prediction, but these differences are very small. Considering different assumptions in the numerical simulations and the theoretical analysis, the discrepancies are understandable, e.g., the lower flow velocity and the higher thermodynamic states are due to a larger increase in entropy in the numerical

Table 3 C-J parameters of the spherically diverging and the planar detonations in a gas mixture of stoichiometric oxygen/ hydrogen with $20 \%$ argon dilution

\begin{tabular}{llll}
\hline & $D(\mathrm{~m} / \mathrm{s})$ & $P_{\mathrm{CJ}} / P_{0}$ & $T_{\mathrm{CJ}}(\mathrm{K})$ \\
\hline Spherical case (this study) & 2249.3 & 17.6 & 3,530 \\
Planar case (this study) & 2350.3 & 18.0 & 3,567 \\
Planar case [13] & 2357.3 & 19.1 & 3,594 \\
\hline
\end{tabular}
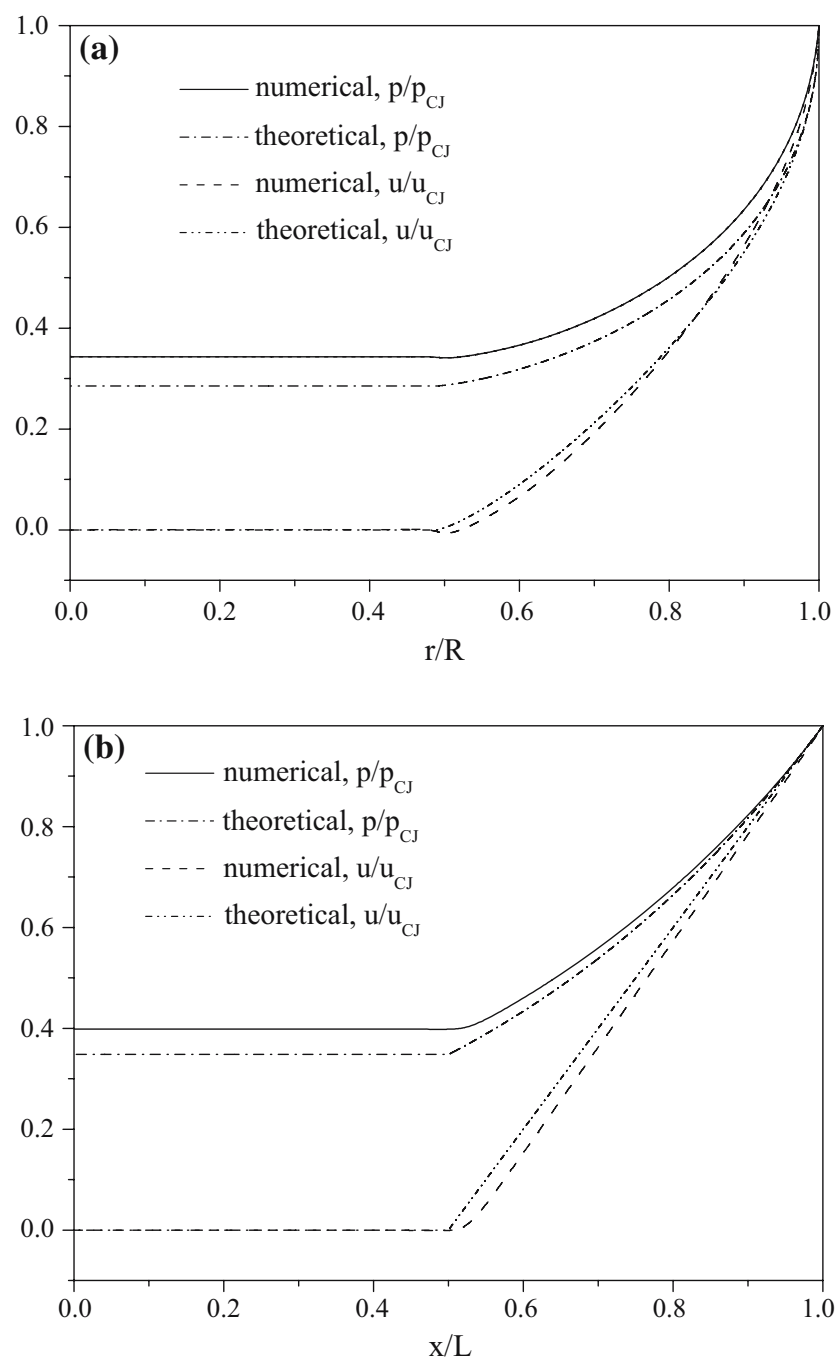

Fig. 2 Comparison between numerical results and the selfsimilar law by showing pressure and velocity distributions behind the detonation front starting from the C-J plane. a Spherically diverging detonation. b Planner detonation

simulation where the detailed chemical reaction is applied. In conclusion, the code is well verified and the numerical solutions are acceptable with reasonable accuracy.

\section{Results and discussion}

For the present numerical investigation, initial conditions are specified as follows: in the region of $r<R$, as shown in Fig. 1, the detonable gas mixture is a stoichiometric hydrogen/oxygen mixture with $25 \%$ argon dilution at initial conditions of $P_{0}=0.02 \mathrm{MPa}, T_{0}=$ $298 \mathrm{~K}$ and $u_{0}=0$; and in the region of $r>R$, the initial detonation profile is specified in a $25 \mathrm{~cm}$ distance in the 
radial direction according to the numerical solution of a planar detonation calculated by using the same gas mixture at the same initial conditions as mentioned above. The detonation front is positioned at $r=R$ and propagates towards the computational domain center. The condition is adopted based on the assumption that the area-converging effect on the spherically converging detonation wave front is negligible at its early stage so that the uncertainness due to the initiation and the deflagration detonation transition (DDT) process in experiments can be eliminated. The grid is equally spaced and the grid size is $0.01875 \mathrm{~cm}$ in length for all the cases. It is known that the numerical post-shock parameters of converging shock waves are sensitive to the computational mesh size, so the grid convergence test was carried out by using two grid sizes of 0.025 and $0.0125 \mathrm{~cm}$, respectively. The comparison shows that the post-shock pressure in the finer grid is about $5 \%$ higher than that in the coarser one when the detonation front reached at $r / R=0.01$, and the difference in the post-shock temperature falls within $8 \%$. Except these post-shock parameters, the pressure and temperatures behind the detonation front in two grid resolutions are almost the same. Therefore, the presently applied grid size does not affect significantly the dynamic characteristics of the converging detonation waves and the tendency of the post-shock parameters variations.

In the following discussion, four major physical issues will be identified, which may interact with each other in different ways. The first issue is the dynamic characteristics of the post-detonation flow during spherical detonation converging process. The second one is the effect of the chemical heat release and gas dissociations on detonation propagation. For this purpose, the spherically converging shock wave is also calculated and compared with the detonation case. The third one is the role of the spherical detonation diameter since it is believed to be a key factor in determining the final flow state near the focal point. The last one is the effect of the initial gas state in the front of the detonation wave on the post-detonation flow parameters during converging process.

\subsection{Propagation of spherically converging detonations}

Figures 3 and 4 present pressure and temperature distributions in the entire computational domain during the converging process. In these figures the non-dimensional flow variables are shown in $z$-direction, and other two directions denote propagating distance and the corresponding elapsed time, respectively. Results of the hydrogen-oxygen detonation are presented in Figs. 3a and $4 \mathrm{a}$, and shock wave ones in air are plotted in Figs. 3b
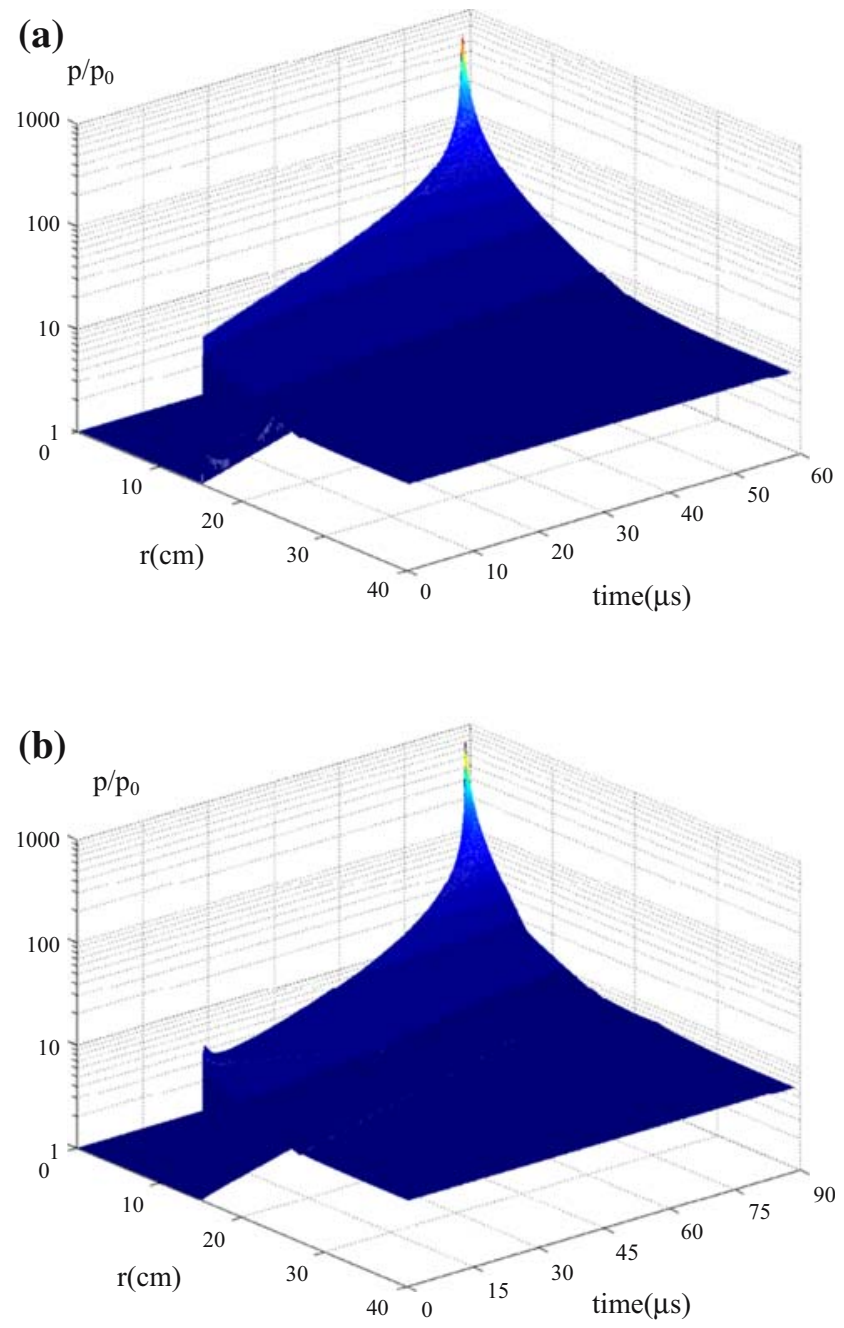

Fig. 3 Time evolution of pressure distributions during the converging processes of spherical shock and detonation. a Spherically converging detonation in a hydrogen-oxygen mixture. b Spherically converging shock wave in air

and $4 \mathrm{~b}$. The computation is terminated at the moment of $t=58 \mu \mathrm{s}$ for the spherical detonation and at $t=88 \mu \mathrm{s}$ for the spherical shock wave. At the moments, two wave fronts arrive at the same position of $r / R=0.01$ and the numerical results show that the maximum temperature is not higher than $6,000 \mathrm{~K}$ for the detonation and less than $7,000 \mathrm{~K}$ for the shock wave, both fall in the prediction limitation of the applied reaction model.

The comparison of Fig. 3a with $3 \mathrm{~b}$ shows that the pressure distributions of both cases vary approximately in a similar way. The detonation pressure distribution appears higher than that of the shock wave at the earlier stage, but they reach to the similar level later when the computation is terminated at $r / R=0.01$. This is because that the leading shock front of the detonation is supported by the chemical reaction heat, while there is no such a driving force for the shock wave. At the later 

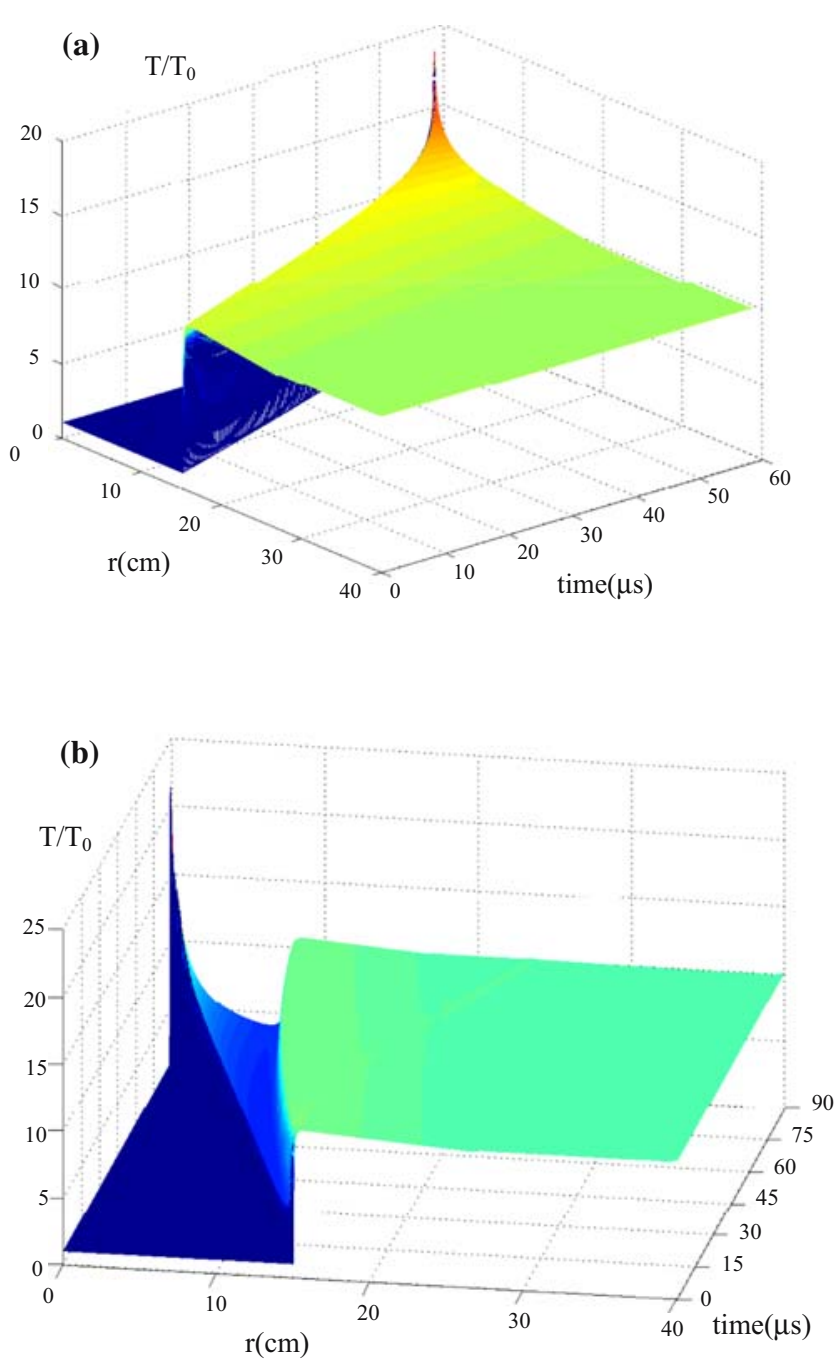

Fig. 4 Time evolution of temperature distributions during spherically converging process. a Spherically converging detonation in a hydrogen-oxygen mixture. b Spherically converging shock wave in air

stage of the converging process, compression mechanism due to the area convergence becomes dominant and the contribution from the chemical reaction heat to the flow state is negligible, thus, the maximum pressures of the two cases arise to a similar level.

Time evolutions of the temperature profiles of two cases are presented in Fig. 4. One of the obvious differences is the discontinuity interface appearing in the shock wave case and propagating towards the focal point. The discontinuity is the contact surface separating the combustion product from the ambient air and moving with the local flow velocity. The post-shock temperature is lower at the beginning of the shock wave converging because of the lack of the chemical energy release. As the wave converges towards the focal point, the leading shock wave gets stronger and stronger, thus resulting in a rapid increase in post-shock temperature up to 24 times higher than the initial temperature. This value is even higher than the post-detonation temperature. The figure also indicates that at the final stage of the converging process the effect of the chemical heat release in detonation is minor and the molecular dissociation becomes dominant. Moreover, the molecular dissociation effect on the flow state is more obvious in the detonation case than in the shock wave because the gas dissociation temperature is higher for air.

\subsection{Gas dissociation effect}

To examine carefully the role of the chemical reaction and gas dissociation in the converging process, the mass fraction profiles of three species at five time steps from the spherically converging detonation are plotted in Fig. 5 and those from the spherical shock wave in air are presented in Fig. 6.

Figure 5a shows that the increase in $H$ mass fraction is first gradually during the detonation converging process, but becomes rapidly as the detonation front propagates near the focal point. The $O$ mass fraction profile in Fig. $5 \mathrm{~b}$ shows the same tenancy and it finally reaches to $28 \%$ of the total mass. This result indicates that most of oxygen in the gas mixture is dissociated, which leads to a low generation rate of $\mathrm{H}_{2} \mathrm{O}$, as show in Fig. 5c. The high $\mathrm{O}$ mass fraction versus low $\mathrm{H}_{2} \mathrm{O}$ mass fraction suggests that the endothermic reaction becomes dominant, while the exothermic reaction gradually diminishes.

Figure 6 shows the mass fractions of $O, N$ and $O_{2}$ in shock converging process. A rapid increase in $O$ mass fraction and reduction in $\mathrm{O}_{2}$ mass fraction take place near the focal point. Finally, the mass fraction of $O$ reaches to $23 \%$, while the most of $\mathrm{O}_{2}$ is dissociated. This is similar to that observed in the detonation case. $N$ is produced at the leading shock front only after the wave front reaches close to the focal point, as shown in Fig. 6b. By comparing the $\mathrm{N}$ mass fraction with $\mathrm{O}$ and $\mathrm{O}_{2}$ in Fig. 6a, c, it is found that the $N_{2}$ dissociation is much later and less than $\mathrm{O}_{2}$ because the dissociation temperature is about $2,500 \mathrm{~K}$ for $O_{2}$ and $4,000 \mathrm{~K}$ for nitrogen. Therefore, the gas dissociation in the converging shock in air is less active than that in the converging detonation of the hydrogen-oxygen mixture. This is the reason why the final temperature near the focal region in the shock converging case can increase faster and higher than the detonation.

In order to further illustrate the dissociation effect in the detonation converging process, calculations are also conducted in which all the chemical reactions are switched off immediately after the computation starts, and $\mathrm{O}_{2}$ and $\mathrm{H}_{2}$ are frozen and act as inert gases without 

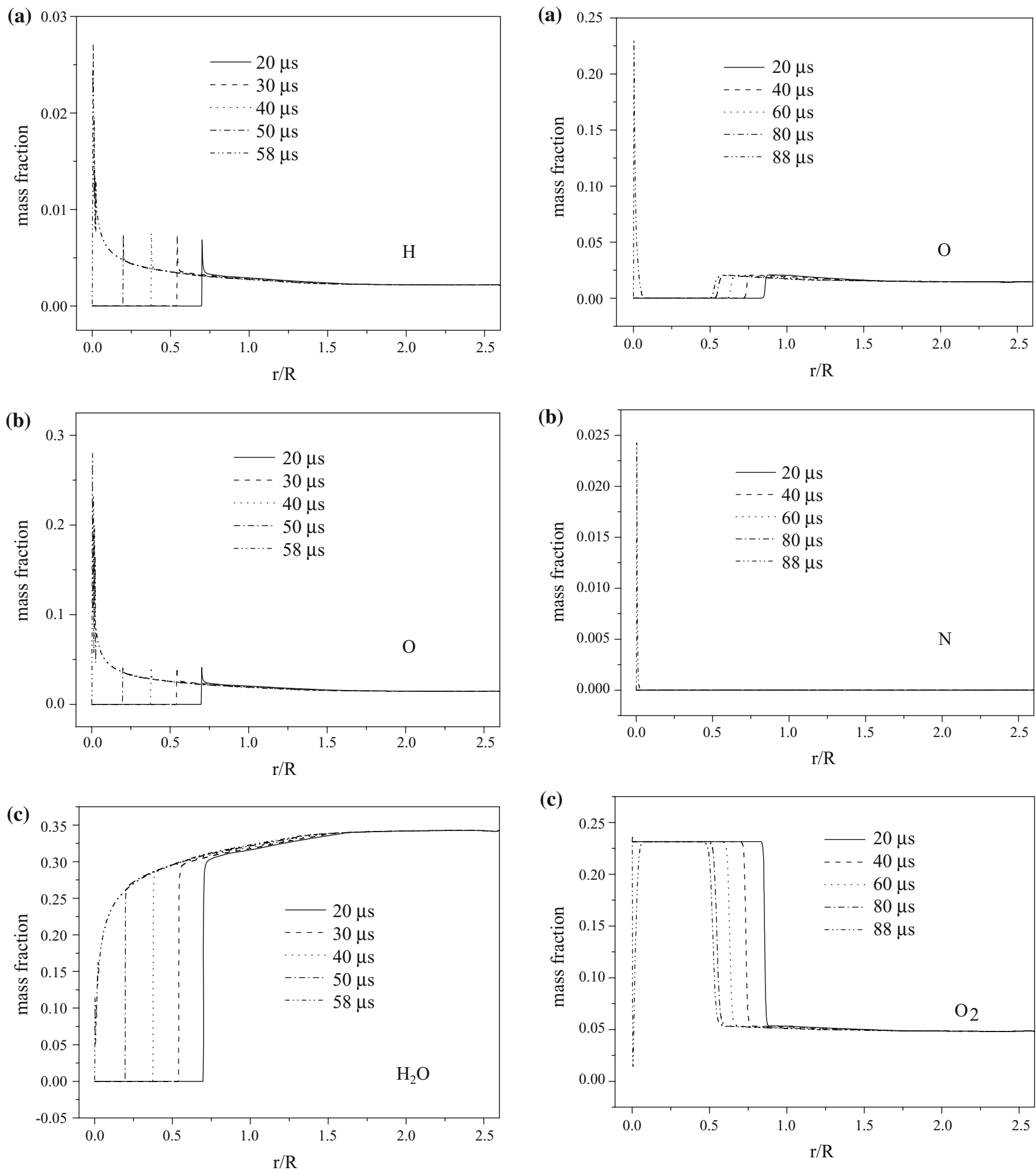

Fig. 5 Mass fraction distributions at a series of time steps during detonation converging in a hydrogen-oxygen mixture: $(20,30,40$, 50 and $58 \mu$ s). a $\mathrm{H} ; \mathbf{b} \mathrm{O} ; \mathbf{c} \mathrm{H}_{2} \mathrm{O}$

chemical reactions nor gas dissociations. As the shock front reaches to the same position as in the two cases investigated above, the maximum post-shock pressure

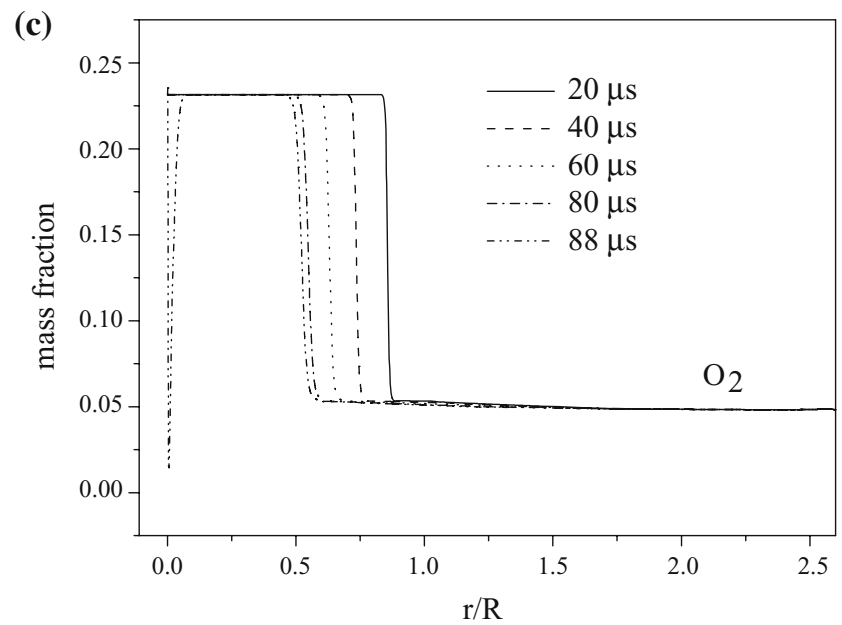

Fig. 6 Mass fraction distributions at a series of time steps during shock wave converging in air: $(20,40,60,80$ and $88 \mu \mathrm{s})$. a $\mathrm{O} ; \mathbf{b} \mathrm{N} ; \mathbf{c ~ O}_{2}$

ratio reaches to about 980 , being almost the same as in the previous two cases, but the maximum post-shock temperature ratio is increased to about 50 and becomes 

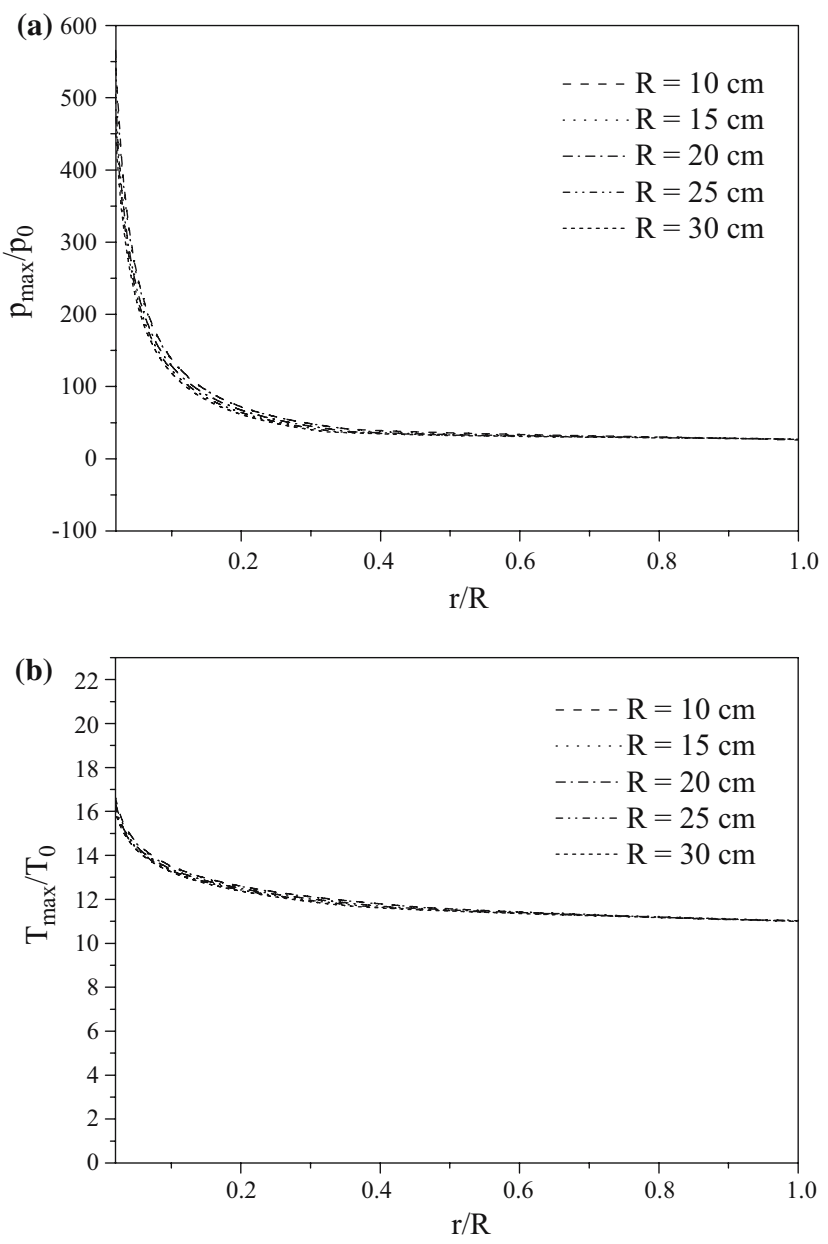

Fig. 7 Maximum values of the post-detonation state parameters of the spherical detonation during its converging in five cases with different initial diameters. a Maximum pressure. b Maximum temperature

twice of that in the converging detonation case. This test case is artificially deigned and cannot be realized experimentally, but does indicate the important role of gas dissociations in detonation converging process.

\subsection{The role of spherical detonation diameters}

It is believed that the post-detonation flow state must be closely related to the diameter of the initial spherical detonation. In order to examine the role of the diameter in the detonation converging, five cases are simulated by specifying the initial detonation diameter to be 20 , $30,40,50$ and $60 \mathrm{~cm}$, respectively, and numerical solutions are plotted together to demonstrate their differences. During these simulations, the maximum pressure $P_{\max }(r)$ and temperature $T_{\max }(r)$ at different time step are selected, recoded according to their locations and presented in Fig. 7.
Table 4 Maximum values of dimensionless pressure and temperature when the spherical detonation is propagating thought three positions

\begin{tabular}{lllllll}
\hline & $P_{\max } / P_{0}$ & \multicolumn{4}{c}{$T_{\max } / T_{0}$} \\
\hline$r / R$ & 0.4 & 0.1 & 0.02 & 0.4 & 0.1 & 0.02 \\
$R=15 \mathrm{~cm}$ & 37.4 & 136.2 & 526.9 & 11.8 & 13.5 & 16.3 \\
$R=30 \mathrm{~cm}$ & 34.5 & 118.2 & 449.5 & 11.6 & 13.2 & 16.0 \\
\hline
\end{tabular}

The dimensionless pressure $P_{\max } / P_{0}$ is plotted in Fig. $7 \mathrm{a}$ and the temperature $T_{\max } / T_{0}$ is in Fig. $7 \mathrm{~b}$. The five cases are simulated at the same initial conditions of $P_{0}=0.02 \mathrm{MPa}$ and $T_{0}=298 \mathrm{~K}$. Figure 7 shows that $P_{\max } / P_{0}$ and $T_{\max } / T_{0}$ curves are in a similar distribution against the dimensionless radius $r / R$ for all the cases. The maximum pressure increases by 500 times at the final stage, as shown in Fig. 7a, and the maximum temperature arises about 16 times. Some minor differences can be distinguished, for example, the pressure and temperature discrepancy vary within 15 and $2 \%$, respectively, when $R$ increases from 15 to $30 \mathrm{~cm}$. The relevant results summarized in Table 4 demonstrates the differences at the same position of $r / R$. The post-detonation state is slightly higher in the case where initial detonation diameters are smaller. Note that the same flow state at a given $r$ (e.g., $1.5 \mathrm{~cm}$ ) for the case of $R=15 \mathrm{~cm}$ is reached at a larger $r$ (i.e., $3 \mathrm{~cm}$ ) for the case of $R=30 \mathrm{~cm}$ for the same ratio of $r / R$. Hence, a much higher flow state can be reached for the later case when the detonation front arrives at $1.5 \mathrm{~cm}$. As a result,the bigger initial detonation diameter, the higher flow state can be achieved near the focal point.

\subsection{Effect of initial conditions on the converging process}

Taking the spherical detonation of a $60 \mathrm{~cm}$ initial diameter as an example, the effect of initial flow states on the converging process is examined. Five cases are also calculated by taking the initial gas pressure to be 0.02 , 0.04, 0.06, 0.08 and $0.1 \mathrm{MPa}$, respectively. The numerical results, selected in the same way as mentioned in Fig. 7, are plotted together in Fig. 8. The maximum pressures $P_{\max } / P_{0}$ against $r / R$ are plotted in Fig. 8 a and the maximum temperatures $T_{\max } / T_{0}$ are in Fig. 8b. The overlapping of the five pressure curves in Fig. 8a suggests that the maximum pressure increases proportionally to the initial pressure. Figure $8 \mathrm{~b}$ shows that the maximum temperature also increases with the initial pressure, but the increasing rate is much less than the maximum pressure. For example, when the spherical detonation front reaches to the position of $r / R=0.02$, the maximum 

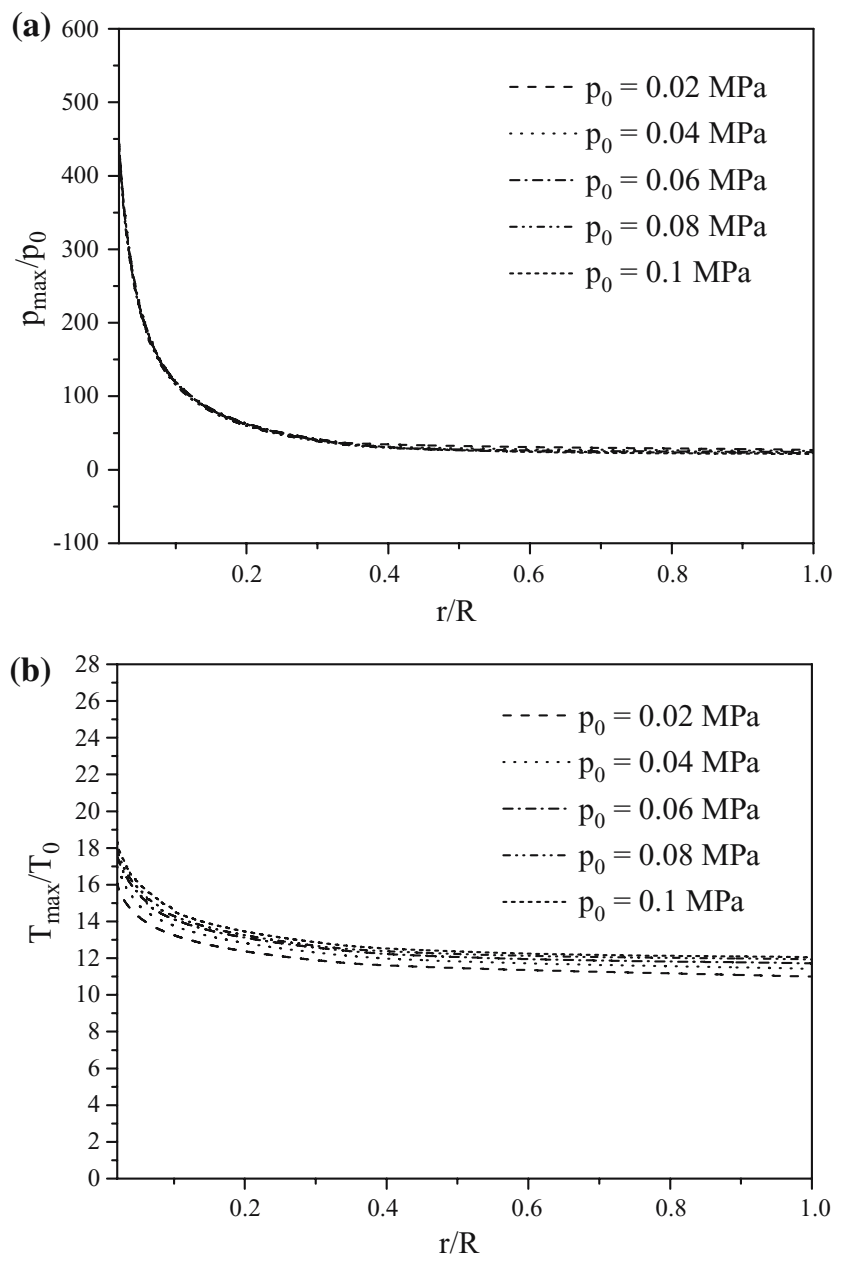

Fig. 8 Maximum values of the post-detonation state parameters of the spherical detonation during its converging in five cases with different initial pressures. a Maximum pressure. b Maximum temperature

pressure increases by about five times, but the maximum temperature rises only from 4,778 to $5,448 \mathrm{~K}$, as the initial pressure varies from 0.02 to $0.1 \mathrm{MPa}$. Therefore, the initial pressure increases the post-detonation pressure dramatically, but its effect on the post-detonation temperature is much less.

\section{Conclusions}

From the discussion above, some concluding remarks can be drawn as follows:

1. The extremely high post-detonation flow state can be achieved in spherically converging detonation because of the chemical reaction heat and the everdecreasing area of the wave front. However, the detonation wave accelerates slower than does a non- reactive shock wave in the same initial strength due to gas dissociations that become dominant in the final stage of the converging process.

2. The chemical reaction heat in the spherically converging detonation helps to sustain the post-detonation flow sate before the wave front reaches to the position around $r / R=0.02$, and the further increase in the post-detonation flow state near the focal point is mainly due to geometry converging.

3. The post-detonation temperature near the focal point mainly depends on gas dissociations, and therefore, the much higher thermal flow sate can be obtained by using the gases having a higher dissociation temperature limit.

4. The dimensionless maximum pressure and temperature with respect to their initial value are approximately scaled with the ratio of the propagation radius over the initial detonation diameter. Hence, an increase in initial detonation diameter results in an increase in maximum pressure and temperature at the same radius.

5. An increase in initial pressure of a detonable gas mixture will make the post-detonation pressure increase proportionally, and also elevate the postdetonation temperature, but in a much slower rate.

\section{References}

1. Lee, J.: Theory of explosion, MERL Report 69-10, 1969

2. Nettleton, M.A.: Gaseous Detonations: Their Nature, Effects and Control. pp. 37-40, Chapman and Hall Ltd, New York (1987)

3. Guderley, G.: Powerful spherical and cylindrical compression shocks in the neighborhood of the center of the sphere and of the cylinder axis. Luftfahrtforsch 19, 302-312 (1942)

4. Li, H., Ben-Dor, G.: A modified CCW theory for detonation waves. Combust. Flame 113, 1-12 (1998)

5. Kogarko, S.M., Adushkin, V.V., Lyamin, A.G.: Nauchno Teknicheskie Problemy Goreniya i Vzryva 2(22) (1965)

6. Bull, D.C., Elsworth, J.E., Hooper, G., Quinnt, C.P.: A study of spherical detonation in mixtures of methane and oxygen diluted by nitrogen. J. Phys. D: Appl. Phys. 9 (1976)

7. Terao, K., Wagner, H.G.: Experimental study on spherically imploding detonation waves. Shock Waves J 1, 27-34 (1991)

8. Terao, K., Akaba, H., Shiraishi, H.: Spherically imploding detonation waves initiated by a two-step divergent detonation. Shock Waves J 4, 187-193 (1995)

9. Jiang, Z., Takayana, K., Chen, Y.S.: Dispersion conditions for non-oscillatory shock capturing schemes and its applications. Computat. Fluid Dyn. J 4, 137-150 (1995)

10. Jiang, Z:: On the dispersion-control principles for nonoscillatory shock capturing schemes, ACTA Mech. Sinica 20(1), 1-15 (2004)

11. McBride, B.J., Zehe, M.J., Gordon, S.: NASA Glenn coefficients for calculating thermodynamic properties of individual species. National Aeronautics and Space Administration Glenn Research Center Cleveland, Ohio, NASA/TP-2002211556 (2002) 
12. Steger, J.L., Warming, R.F.: Flux vector splitting of the inviscid gasdynamics equations with applications to finite difference methods. J. Comp. Phys. 40, 263-293 (1981)

13. Schultz, E., Shepherd, J.: Validation of detailed reaction mechanisms for detonation simulation. Graduate Aeronautical Laboratories, California Institute of Technology, Pasadena, Ca 91125 (2000)
14. Li, W.X.: One-dimensional nonsteady flow and shock waves, pp. 466-467, National Defence Industry press, Peking (2003)

15. Chang, L.N., Zhan, D.L., Hu, Z.M., Jiang, Z.: An extension of dispersion-controlled dissipative scheme and its application in simulating detonation Mach reflection. Chin. J. Computat. Phys. 22, 189-196 (2005) 